\title{
A Novel Approach in Analyzing Traffic Flow by Extreme Learning Machine Method
}

\author{
Yasin SÖNMEZ, Hüseyin KUTLU, Engin AVCI
}

\begin{abstract}
The objective of this study is to detect abnormal behaviours of moving objects captured in highway traffic flow footages, classify them by using artificial learning methods, and lastly to predict the future thereof (regression). To this end, the system being the object of the design and application consists of three stages. In the first stage, to detect the moving object in the video, background/foreground segmentation method of Mixture of Gaussian (MOG), and to track the moving object, Kalman Filter-Hungarian algorithm method have been used. In the second stage, by using the coordinates of the object, such details as location, distance in terms of time, and speed of the object are obtained, and by using total pixel count data relating to the shape of the object are obtained. The software based on the specifically elaborated algorithm compares these data with the data in the table of rules set down for the road under surveillance, and generates an attribute table comprising anomalies of the objects in the video. In the last stage, however, the data included in the attribute table have been classified and predictions by the artificial learning method, Extreme Learning Machine (ELM) made.
\end{abstract}

Keywords: anomaly classification and prediction (regression); artificial learning; Extreme Learning Machine (ELM); traffic flow video analysis

\section{INTRODUCTION}

Classical video surveillance methods based on human surveillance and inter-city and intra-urban road traffic surveillance systems do not suffice for reasons such as instant evaluation, continuous updating of information, high amount of data and manual warning [1-4]. The ability to obtain meaningful and interpretable data from video footages with autonomous systems in today's technology has reached an easier and more effective level with the developing hardware structure and software blocks $[1,2,5$, 8]. This level of technology in this area has reduced the margin of human error in visual evaluation, and instead, increased the computer control and accuracy of data. With this state of improvement and development, advanced technological surveillance systems have begun to be used instead of classical techniques to watch over and inspect intercity and intra-city road traffic. A considerable part of these advanced technological techniques have been generated from autonomous systems operating on artificial intelligence methods [12].

Analysis of highway traffic flow footage may be defined as classification of anomaly described as illegal behaviour by detection and tracking of moving objects in the video streams from recording device hardware, and prediction thereof [9-12]. Systems consisting of hardware and software enabling autonomous monitoring of the traffic flow provide us with assessable and interpretable information. With this information, it is aimed to ensure the security of traffic flow. Collection of these data by computer-aided autonomous systems reduces the margin of human error and increases computer control and the accuracy of the data in the assessment. This, in turn, yields high-performance warning systems for use in highway traffic which is monitored for security [11-15]. The objective of this study is to detect abnormal behaviours of moving objects from highway traffic flow footage, and to classify them and make a prediction of future state thereof by using artificial learning methods. Anomalies in traffic videos refer to detection of violations by moving objects (vehicles or pedestrians) of rules established for the road being monitored. Prediction of future states, on the other hand, is numerical expression of whether behaviour and situation of detected objects can be assessed as anomaly.

\subsection{Related Works}

Cheung and Chandrika (2004) compared the background subtraction algorithms used to detect moving vehicles and pedestrians in video data obtained from urban highway traffic. As a consequence of this comparison, they have shown that problems encountered in detection methods will be overcome upon introduction of robust and sophisticated methods by making use of variations of such simple techniques as frame difference and median filtering [12].

Morris and Trivedi (2008) identified the types of, and classified the vehicles they tracked in highway traffic flow videos, and they were successful in their efforts by $94 \%$. They also recorded the statistical data regarding the hours of use of the highway by the vehicles they had classified. In their upcoming study, they classified vehicle trajectories by using various clustering algorithms and elicited models of the highway [13].

Çandar and Gerek (2014) have designed an expert system that detects violations such as faulty lane change, close follow-up and over speeding by methods based on differential and zone-based motion analysis, and generates an alarm relating to these detections [14].

Öz and Görgünoğlu (2016) have provided information about detection of anomalies via video surveillance systems under four sub-headings: detection and surveillance of anomalies, feature extraction, training and learning as well as modelling and classification. In the light of these data, they have concluded that anomaly detection studies are spread over broad area including such as targets, monitored area, and level of resolution and that systems establishing general performance criteria that encompass all areas have yet to emerge [15].

In this study, a new video analysis system relying upon the artificial learning method, The Extreme Learning Machine (ELM), which detects and classifies anomalies of moving objects in highway traffic flow videos and makes predictions as to their future states, has been proposed. This system comprises MOG as the background/foreground segmentation method for detection of moving objects in videos and Kalman Filter-Hungarian algorithm methods for tracking thereof, and classification of anomalies using ELM method by the software generating the attribute table containing data such as location, duration, speed, shape and the relevant anomaly. 
below:

Characteristics and novelties of this method are given

- For detection of moving objects in videos, performances of 7 different methods (Basic Motion Detection, One Gaussian (1-G), Gaussian Mixture Model (GMM), Kernel Density Estimation (KDE) Codebook (CBRGB), Eigen Backgrounds (Eigen), Mixture of Gaussians) have been comparatively evaluated and assessed. Mixture of Gaussians which is the highest performing method for fixed background has been used.

- To generate the attribute table of the moving object based on coordinate data, software based on specific algorithms has been elaborated.

- For detection and classification of anomalies as well as prediction of future states based on attribute data, classic ELM which is a high performing artificial learning method, has been selected. In order for the classical ELM performance to be high and the training period to be short, automatic optimization has been developed using the crossover method to ensure the best combination of hidden layer count and activation function.

Organization of other parts of this article is given as follows. Materials and the proposed method, experiments of the proposed method, conclusion and discussion are presented in section 2, 3, 4 and 5 respectively.

\section{MATERIALS}

The materials used in the three-stage method proposed for solving the problem being the object of this study are examined in this section. At the first stage, the background/foreground segmentation method, Mixture of Gaussians (MOG), and the Kalman Filter-Hungarian algorithm methods for object-trajectory estimationassignment were used. In the second stage, the algorithm of the software that generates the attribute table, and in the third stage, the ELM method used for classifying the anomaly and estimating the future states are explained.

\subsection{Video Analysis}

The video analysis, also referred to as fast image flow (30 images at a time), begins with the detection of the movement of the object. Motion detection is based on the principle that the moving region is separated from other regions in the image.

According to this principle, separation and differentiation of the motion from the background is done by comparing pixel differences at the same coordinates of the examined image with the reference image, either with a predetermined threshold value or with a statistical approach to these differences $[1,2,8,11,12]$.

\subsubsection{Mixture of Gaussians}

To obtain one pixel the MOG (mixture of Gaussian) is used as a density function. The location of each pixel is defined in Eq. (1) and each pixel is indicated as a number of Gaussian function that forms a probability distribution function $f\left(I_{t}=u\right)$. In Eq. (1) $x$ is the probability of a certain pixel at time $t$.

$$
f\left(I_{t}\right)=u=\sum_{i=1}^{K} \omega_{i, t} \cdot n\left(x_{t}-\mu_{i, t}, \sum i, t\right)
$$

Each of the $K$ Gaussian distributions is deemed to describe only one of the observable background or foreground objects. In practical cases, $K$ is set to be between 3 and 5 . The mean $x$ of each Gaussian function can be thought of as an educated guess of the pixel value in the next frame. $\omega_{i, t}$ is an estimate of the weight of the $i^{\text {th }}$ Gaussian in the mixture and $u_{i, t}$ is the mean value of the $i^{\text {th }}$ Gaussian in the mixture, at time $t$ [12]. Since MOG is a parametric model, it can be updated adaptively without holding large video frames in memory. This method is known for its ease of use as algorithm and application, as well as the small amount of processing load. The background is only tied to the previous frame, which allows it to give better results as compared to other methods in case of presence of noise arising out of environmental effects, and for adaptation when there is a change of background. The reason why the method is used in the analysis of highway traffic flow videos is that it offers a powerful solution in static background videos [16].

\subsubsection{Kalman Filter}

In tracking linear dynamical systems that have Gaussian noise the Kalman filter is an effective technique. The prediction and correction are crucial parameters for the Kalman filter. To predict the new state of the variables in state model prediction step is deployed:

$$
\begin{aligned}
& \bar{X}^{t} v=D X^{(t-1)}+W \\
& \bar{\Sigma}^{t}=D \Sigma^{(t-1)} D^{\mathrm{T}}+Q^{t}
\end{aligned}
$$

where $\bar{X}^{t}$ and $\bar{\Sigma}^{t}$ are the state and the covariance predictions at time $t$. $D$ is the state transition matrix which defines the relation between the state variables at time $t$ and $t-1$. $Q$ is the covariance of the noise $W$.

\subsubsection{Hungarian Algorithm}

The Hungarian algorithm is essentially a method presented in relation to the assignment problem. The goal of the algorithm is to achieve the maximum match. Matching problems can also be considered as a graph problem in general. That is, the intended mapping process can be modelled with a graph, and all the possibilities provided by graph theory can be used on this model. After the trajectory estimation of the moving object detected by MOG in a video is performed by the Kalman filter, this trajectory assignment (matching) is performed by the Hungarian algorithm.

\subsection{Artificial Learning}

The generally common characteristic of the artificial learning methods is that they can classify the data about the problem on hand (classification of output data of the input data) and predict (predicting the class of future input data 
based on output data). The artificial learning method utilized in this study is the Extreme Learning Machine which is a feed-forward Artificial Neural Network (ANN) model with a single hidden layer (ELM) [18-20].

\subsubsection{Extreme Learning Machine (ELM)}

ELM is a learning approach developed for feedforward, single hidden layered ANNs. Unlike the ANN where coefficients are renewed on the basis of gradient in learning processes, in this model while input weights are randomly selected, the output weights are analytically calculated. An analytical learning process significantly reduces both solution time and the probability that an error value can be entrapped in a local minimum. Just as a linear function can be selected to activate the cells in the hidden layer of ELM, so it is possible to use non-linear activation functions (such as sigmoid, sinus, Gaussian). Furthermore, non-differentiable or intermittent activation functions can also be used in these cells. Fig. 1 shows the structure of a feed-forward ANN trained by ELM. Eq. (3) can be calculated as follows [19].

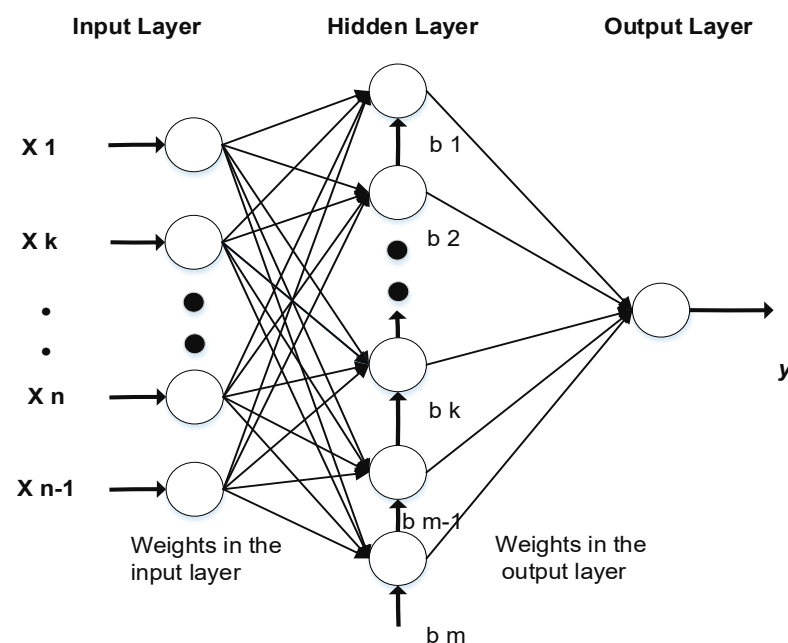

Figure 1 The Schematic diagram of single hidden layer feed forward neural network

$y=\sum_{i=1}^{m} \beta_{i} g\left(\sum_{i=1}^{n} w_{i, j} x_{i}+b_{i}\right)$

$x_{i}$ indicates input, $y$ identifies the output, where $m$ and $n$ are the numbers of neurons in the hidden and input layers, respectively.

In Eq. (3) $w_{i, j}$ is input weights, $\beta_{i}$ is output weights, $\mathrm{b}_{\mathrm{j}}$ is biases of the neurons in the hidden layer and $g(\cdot)$ is the activation function. The learning mechanism of ELM is modelled to achieve zero error averages of the training data set by $\sum_{i=1}^{N}\left\|y_{j}-t_{j}\right\|=0$. Where $t$ is desired output and $N$ shows the number of instances in training dataset.

\section{THE PROPOSED METHOD}

In this study, a new video analysis system based on the artificial learning method, Extreme Learning Machine, which detects and classifies the anomalies and predicts the future state of moving objects in highway traffic flow videos, has been proposed. The proposed method consists of hardware and software phases. A traffic surveillance camera has been used as hardware. The software phases of the system are described in the following sections. Block diagram of the proposed system is shown in Fig. 2.

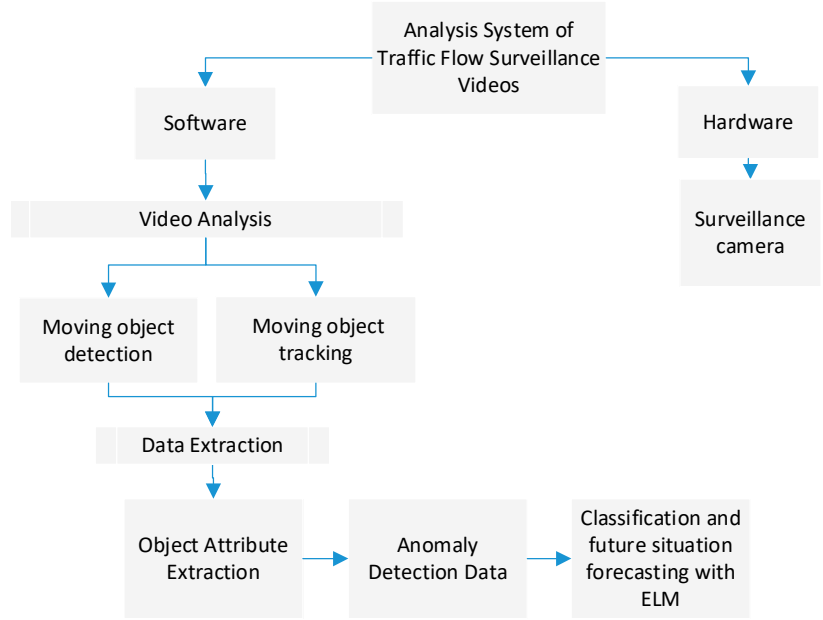

Figure 2 Block diagram of the proposed method

Stage 1: The environment (road) to be analysed where there is traffic flow, and moving vehicles or objects are found - is monitored and the footage is recorded by a camera from a fixed point for a certain period of time.

Stage 2: Video records are segmented into pieces of appropriate formats and equal durations by preliminary processing phase.

Stage 3: The Prepared pieces are transferred into the software for detecting and tracking moving objects in videos codified by $\mathrm{C}++$ language in the Open $\mathrm{CV}$ program. As was previously explained in section 2.1 of this article, this software runs MOG for background/foreground subtraction to detect the object, and then the Kalman filter which predicts the trajectory of the moving object, and thereafter the Hungarian algorithm which makes an assignment to the predicted point.

Fig. 3 shows a diagram of moving object detection and tracking steps in the video analysis of the proposed method.

Stage 4: The points through which the object has passed in the previous stage during the tracking are recorded in the file (in terms of $x$ and $y$ coordinates on the analytical plane).

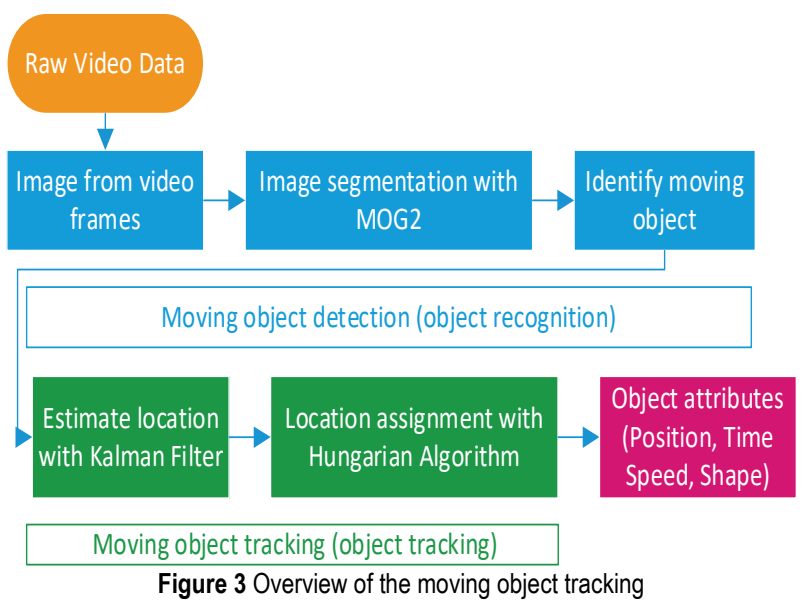

Stage 5: Based on the coordinate values of the object an attribute table is generated. The attributes are prepared 
using the characteristics provided in Tab. 1 by the software the pseudo code which is given below. data.

Algorithm 1: Pseudo code of the object attributes

Input: Data from object tracking - $\mathrm{x}, \mathrm{y}$ coordinates $)$

Output: Object attribute

$1: \mathrm{n}=$ numberofobject;

2: $\mathrm{OID}=$ ObjectID

3: fori $=1$ to ndo

4: $\mathrm{Lcv}-\mathrm{Fcv}=\operatorname{Dom}(\mathrm{OID}(\mathrm{n}))$

4: $\mathrm{Lcv}-\mathrm{Fcv}=\operatorname{Sub}(\mathrm{OID}(\mathrm{n}))$

5: $\mathrm{Ic}=\mathrm{L}(\mathrm{OID}(\mathrm{n}))$

6: $(\mathrm{Lcv}-\mathrm{Fcv}) /$ Time $=\operatorname{Spe}(\mathrm{OID}(\mathrm{n}))$

$7: \operatorname{Tpv}=\operatorname{Sha}(\operatorname{OID}(\mathrm{n}))$

8: endfor

Stage 6: The software whose pseudo code is provided herein below detects the anomaly by comparing data relating to the attributes of the object (Tab. 1) and data related to the rules laid down for the road (Tab. 2).

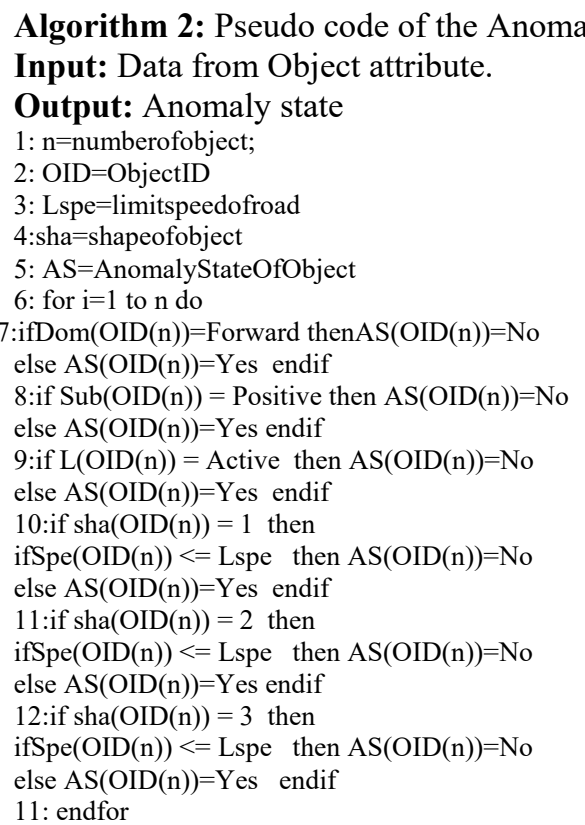

Table 1 Object attribute

\begin{tabular}{|l|c|}
\hline \multicolumn{1}{|c|}{ Data obtained by video analysis } & Attribute \\
\hline $\begin{array}{l}\text { Last coordinate value(Lcv) - First coordinate } \\
\text { value(Fcv) }\end{array}$ & $\begin{array}{c}\text { Direction of } \\
\text { movement }\end{array}$ \\
\hline $\begin{array}{l}\text { Last coordinate value(Lcv) - First coordinate } \\
\text { value(Fcv) }\end{array}$ & Substitution \\
\hline Instant Coordinate value(Ic) & Location \\
\hline $\begin{array}{l}\text { (Last coordinate value - First coordinate value) / } \\
\text { Time (number of frame) }\end{array}$ & Speed \\
\hline Total pixel value(Tpv) & Shape \\
\hline
\end{tabular}

Table 2 Anomaly detection rules

\begin{tabular}{|l|l|c|}
\hline \multicolumn{1}{|c|}{ Attribute } & $\begin{array}{c}\text { Data Conversion } \\
\text { Result }\end{array}$ & $\begin{array}{c}\text { Anomaly State } \\
\text { (AS) }\end{array}$ \\
\hline Direction of movement(Dom) & Forward - Rear & No -Yes \\
\hline Substitution(Sub) & Positive-Negative & No -Yes \\
\hline Location(L) & Active - Stable & No -Yes \\
\hline Speed(Spe) & $\begin{array}{l}<\text { limit - >limit } \\
\text { (Lspe) }\end{array}$ & No -Yes \\
\hline Shape(Sha) & $\begin{array}{l}<\text { limit - >limit } \\
\text { (Lsha) }\end{array}$ & No -Yes \\
\hline
\end{tabular}

Stage 7: Following the detection of the anomaly of the object, ELM method is used to classify the objects under video flow based on their situation of anomaly, and for prediction of states of the objects, on the other hand, ELM Regression method is used. Classification and state prediction (regression) is carried out via ELM, with the data generated after running the said software whose pseudo code is furnished being the input data whereas anomalies being the output data.

\section{EXPERIMENTAL RESULTS}

Experimental results and performances of the proposed method are examined in three stages. In the first stage, number of objects (vehicles and pedestrians) and object detection performances obtained by using MOG2 for background/foreground segmentation and the Kalman Filter-Hungarian algorithm method for object trajectory prediction-assignment are shown in Tab. 3. Fig. 4 shows a representative frame from the trial video [Dataset 1] used for detection. For classification and prediction (regression) used dataset occurred 52 moving object videos. Training and test data were obtained by cross folding on the same dataset

Table 3 Number of objects detected and types

\begin{tabular}{|c|c|l|c|c|}
\hline Object in the video & Piece & $\begin{array}{c}\text { Detection of } \\
\text { Software (Object } \\
\text { Class) }\end{array}$ & Piece & Success \\
\hline $\begin{array}{c}\text { Vehicle } \\
\text { (Automobile, SUV, } \\
\text { Pickup) }\end{array}$ & 12 & Small Vehicle (1) & 12 & $100 \%$ \\
\hline Bus, Truck & 2 & Large Vehicle (2) & 2 & $100 \%$ \\
\hline Pedestrian & 5 & Pedestrian (3) & 5 & $100 \%$ \\
\hline Moving object & 0 & Unknown (0) & 0 & $100 \%$ \\
\hline
\end{tabular}

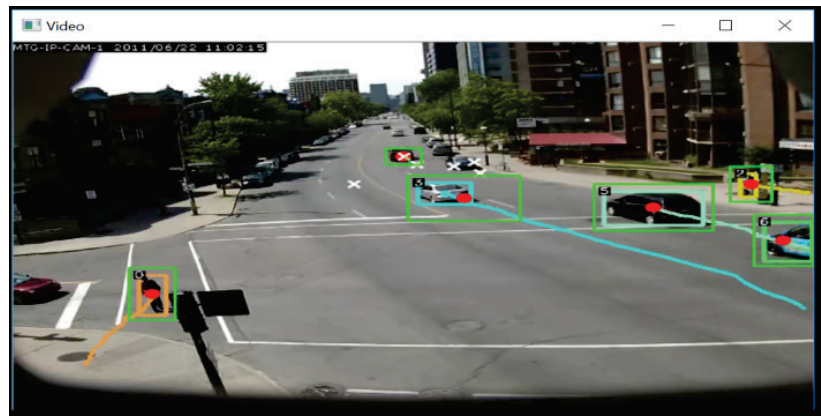

Figure 4 An exemplary framework of experimental videos used in the study

In the second stage, attribute data of the objects are generated as shown in Tab. 4A by using the software. Anomaly detection performed by comparing these data and the rules (Tab. 4B) set down for the road being monitored is provided in Tab. 5. In this stage, our software has detected anomaly of each object with an accuracy rate of $100 \%$

In the third stage, classification and prediction (regression) was carried out by ELM method with the data presented in Tabs. 4A, 4B and 5 being used as input data and emerging anomalies being used as output data. Moreover, in order to compare performance of the ELM method, given in Tabs. 7 and 8 are the results obtained by using the same data in such methods as Support Vector Machine (SVM) and Naive Bayes (NB) for classification, and Support Vector Regression (SVR) and Linear Regression for prediction. These results were obtained by using MATLAB 2103b software and a PC with Intel i7$6500 \mathrm{CPU}$ and 8 GB RAM. 


Table 4A Objects data
\begin{tabular}{|c|c|c|c|c|c|}
\hline $\begin{array}{c}\text { Obj } \\
\text { ID }\end{array}$ & $\begin{array}{c}\text { Obj } \\
\text { Class }\end{array}$ & $\begin{array}{c}\text { Direction of } \\
\text { movement }\end{array}$ & Substitution & Location & $\begin{array}{c}\text { Speed } \\
\mathrm{km} / \mathrm{h}\end{array}$ \\
\hline 1 & 0 & Forward & Positive & Active & 1 \\
\hline 14 & 1 & Forward & Positive & Active & 77 \\
\hline 21 & 1 & Forward & Positive & Active & 63 \\
\hline 30 & 1 & Forward & Positive & Active & $\mathbf{9 4}$ \\
\hline 34 & 1 & Rear & Positive & Active & 33 \\
\hline 46 & 2 & Forward & Positive & Active & $\mathbf{8 1}$ \\
\hline 53 & 2 & Forward & Positive & Active & $\underline{\mathbf{6 8}}$ \\
\hline
\end{tabular}

Table 4B Data for road rule

\begin{tabular}{|c|c|c|c|c|}
\hline $\begin{array}{c}\text { Obj } \\
\text { Class }\end{array}$ & $\begin{array}{c}\text { Direction of } \\
\text { movement }\end{array}$ & Substitution & Location & Speed km/h \\
\hline $\begin{array}{c}\text { Pedestrian } \\
(3)\end{array}$ & Forward & Positive & Active & 1 \\
\hline $\begin{array}{c}\text { Large Vehicle } \\
(2)\end{array}$ & Forward & Positive & Active & $15<X<51$ \\
\hline $\begin{array}{c}\text { Small Vehicle } \\
(1)\end{array}$ & Forward & Positive & Active & $15<X<90$ \\
\hline
\end{tabular}

Table 5 Results of anomaly

\begin{tabular}{|c|c|c|}
\hline $\begin{array}{c}\text { Object } \\
\text { ID }\end{array}$ & $\begin{array}{c}\text { Anomaly } \\
\text { Situation }\end{array}$ & Explanation of anomaly \\
\hline 1 & No & - \\
\hline 14 & No & - \\
\hline 21 & No & speed violation \\
\hline 30 & Yes & direction violation \\
\hline 34 & Yes & speed violation \\
\hline 46 & Yes & speed violation \\
\hline 53 & Yes & \\
\hline
\end{tabular}

In the obtained results number of hidden layers is 50 and activation functions are linear. The test data were taken from a video with 53 moving objects. Test and training has been done from the same data made fold.

Learning parameters pertaining to ELM network are presented in Tab. 6 .

Table 6 ELM learning parameters

Number of neurons in hidden layer:

$2,5,10,20,25,50,75$

Activation Functions: sigmoid (sig), sine (sin), hard limit

(hardlim), triangular basis (tribas), radial basis (radbas) and linear (lin)

Table 7 Performance results of classification methods

\begin{tabular}{|c|c|c|c|c|}
\hline Method & $\begin{array}{c}\text { Training } \\
\text { Achievement }\end{array}$ & $\begin{array}{c}\text { Test } / \\
\text { TP Rate }\end{array}$ & $\begin{array}{c}\text { Training } \\
\text { Time }\end{array}$ & Test Time \\
\hline ELM & $\mathbf{1 0 0 \%}$ & $\mathbf{9 7 , 9 2 \%}$ & $\mathbf{0 , 0 0 5 2 s e c}$ & $\mathbf{0 , 0 0 5 2 s e c}$ \\
\hline NB & $100 \%$ & $86,53 \%$ & $0,01 \mathrm{sec}$ & $0,01 \mathrm{sec}$ \\
\hline SVM & $100 \%$ & $84,61 \%$ & $0,02 \mathrm{sec}$ & $0,02 \mathrm{sec}$ \\
\hline
\end{tabular}

To determine and establish the performances of ELM and other machine learning algorithms, such criteria as Root Mean Square Error (RMSE) and the Mean Absolute Error (MAE) whose equations are given below were used $[21,22]$. The common characteristic of all these criteria is that they describe the error between the estimated error and the actual value.

Mean Absolute Error: $M A E=\frac{1}{n} \sum_{i=1}^{n}\left|X o_{i}-X p_{i}\right|$

Root Mean Square Error: $R M S E=\sqrt{\frac{\sum_{i=1}^{n}\left(X o_{i}-X p_{i}\right)^{2}}{n}}$

where $X o$ is observed values and $X p$ is modelled values.
Table 8 Performance results of regression methods.

\begin{tabular}{|c|c|c|}
\hline \multirow{2}{*}{ Method } & \multicolumn{2}{|c|}{ Performance Indices } \\
\cline { 2 - 3 } & $M A E$ & $R M S E$ \\
\hline ELM & $\mathbf{2 2 , 3 0}$ & $\mathbf{3 6 , 0 7}$ \\
\hline SVR & 33,73 & 42,07 \\
\hline LinearReg & 34,73 & 37,55 \\
\hline
\end{tabular}

\section{CONCLUSION AND DISCUSS}

Classical video surveillance methods based on human surveillance and intercity and intra-urban road traffic surveillance systems do not suffice for reasons such as instant evaluation, continuous updating of information, high amount of data and manual warning. Classification of anomalies described as anomalous behaviour and future state prediction by computer-aided autonomous systems reduces the margin of human error, and increases computer control and data accuracy. This, in turn, provides high performing warning systems in ensuring security and safety of traffic flow. In this study, a new video analysis and interpretation method based on the artificial learning method ELM which will contribute to improvement of autonomous warning systems has been proposed. This method consists of 7 stages which are video recording, video preparation, moving object detection and tracking, recordings of coordinates of the object, generating an attribute table, anomaly detection and classification and prediction by ELM (regression). Performance of the study has been evaluated in three stages in experimental studies. In the first stage, detection and tracking of moving objects (vehicles and pedestrians) from highway traffic flow footages have been achieved with a success rate of $100 \%$. This performance demonstrates suitability and fitness of the methods (MOG, Kalman Filter, and Hungarian Algorithm) used for detection of objects from traffic flow footages. In the second stage, our software used in the method proposed for detection of anomalies shows a success rate of $100 \%$ in detection.

Error-free results have been obtained in the process of extracting interpretable information and data from video analysis by our software used in the proposed method. In the third stage, on the other hand, success rates of the results of classification of objects according to their anomaly, and prediction (regression) by ELM method based on attribute data are $100 \%$ in the training process and $97.92 \%$ in tests.

Its rate of success in prediction of anomaly detection is 22.30 according to MAE error parameter and 36.07 according to RMSE. When experimental results are assessed with regard to classification and future prediction, it is seen that ELM method classifies the data entered into a trained ELM network, as that in a training set designed for detection of anomalies of moving objects (vehicles, pedestrians) from traffic flow footages, with an accuracy rate of $97.92 \%$, and can accurately predict whether this is an anomaly based on the selected error parameter. When evaluating the tests conducted in the CCTV (Closed Circuit Television) monitoring centers, which are the classical monitoring systems, it has been detected that if two or more monitors were monitored continuously for 12-minutes then the security guards missed $45 \%$ of the activity on the screen, and after 22 minutes the performance of activity detection was limited to only $5 \%$. When the results of the proposed method are compared with the classical system, 
it can be observed that a $97.92 \%$ success has been achieved independent of the time.

Table 9 Literature comparison

\begin{tabular}{|c|c|}
\hline Manuscript & Method name \\
\hline $\begin{array}{c}\text { Learning Object Motion Patterns } \\
\text { for Anomaly Detection and } \\
\text { Improved Object Detection [23] }\end{array}$ & $\begin{array}{c}\text { Pixel level probability density } \\
\text { functions (pdfs) }\end{array}$ \\
\hline $\begin{array}{c}\text { Context-Aware Activity } \\
\text { Recognition and }\end{array}$ & $\begin{array}{c}\text { Structural model- mathematical } \\
\text { framework }\end{array}$ \\
Anomaly Detection in Video [24] & \\
\hline
\end{tabular}

In a couple of years, unfavourable impacts of traffic flow and many factors associated therewith will become surmountable thanks to ever advancing hardware and software technology in our globalising and ever more crowded world. Many systems comprising big data as in traffic flow and based on human visual evaluation will give way to and be replaced by systems capable of learning the model of operating autonomously and giving warning when necessary as well as making predictions about the future. In this study, a new system capable of performing the previously mentioned has been proposed as a new approach in traffic flow data analysis by an artificial learning method.

\section{REFERENCES}

[1] Jodoin, J.-P., Bilodeau, G.-A., \& Saunier, N. (2014). Dataset 1: Urban Tracker: Multiple Object Tracking in Urban Mixed Traffic. Accepted for IEEE Winter conference on Applications of Computer Vision (WACV14), Steamboat Springs, Colorado, USA, March 24-26. https://doi.org/10.1109/WACV.2014.6836010

[2] Jodoin, J. P., Bilodeau, G. A., \& Saunier, N. (2014). Urban Tracker: Multiple object tracking in urban mixed traffic. In 2014 IEEE Winter Conference on Applications of Computer Vision, WACV 2014, 885-892. https://doi.org/10.1109/WACV.2014.6836010

[3] Mohan, A. S. \& Resmi, R. (2014). Video image processing for moving object detection and segmentation using background subtraction. 2014 First International Conference on Computational Systems and Communications (ICCSC), 26(5), 288-292. https://doi.org/10.1109/COMPSC.2014.7032664

[4] Zhu, Y., Nayak, N. M., \& Roy-Chowdhury, A. K. (2013). Context-Aware Activity Recognition and Anomaly Detection in Video. Selected Topics in Signal Processing, IEEE Journal of, 7(1), 91-101. https://doi.org/10.1109/JSTSP.2012.2234722

[5] Basharat, A., Gritai, A., \& Shah, M. (2008). Learning object motion patterns for anomaly detection and improved object detection. In Computer Vision and Pattern Recognition, 2008. CVPR 2008. IEEE Conference on, 1-8. https://doi.org/10.1109/CVPR.2008.4587510

[6] Chong, Y. \& Tay, Y. (2015). Modeling Representation of Videos for Anomaly Detection using Deep Learning: A Review. arXiv Preprint arXiv:1505.00523. Retrieved from http://arxiv.org/abs/1505.00523

[7] Brand, M. \& Kettnaker, V. (2000). Discovery and segmentation of activities in video. IEEE Transactions on Pattern Analysis and Machine Intelligence, 22(8), 844-851. https://doi.org/10.1109/34.868685

[8] Chandola, V., Banerjee, A., \& Kumar, V. (2009). Anomaly detection: A survey. ACM Comput. Surv., 41(3), 1-58. https://doi.org/10.1145/1541880.1541882

[9] Tseng, B. L., Lin, C. Y., \& Smith, J. R. (2002). Real-time video surveillance for traffic monitoring using virtual line analysis. In Multimedia and Expo, 2002. ICME'02. Proceedings. 2002 IEEE International Conference on (Vol. 2, 541-544. https://doi.org/10.1109/ICME.2002.1035671

[10] Bas, E., Tekalp, A. M., \& Salman, F. S. (2007). Automatic Vehicle Counting from Video for Traffic Flow Analysis. 2007 IEEE Intelligent Vehicles Symposium, 392-397. https://doi.org/10.1109/IVS.2007.4290146

[11] Batapati, P., Tran, D., Sheng, W., Liu, M., \& Zeng, R. (2014, June). Video analysis for traffic anomaly detection using support vector machines. In Intelligent Control and Automation (WCICA), $11^{\text {th }}$ World Congress on, IEEE, 55005505. https://doi.org/10.1109/WCICA.2014.7053655

[12] Buch, N., Velastin, S. A., \& Orwell, J. (2011). A Review of Computer Vision Techniques for the Analysis of Urban Traffic. IEEE Transactions on Intelligent Transportation Systems, 12(3), 920-939. https://doi.org/10.1109/TITS.2011.2119372

[13] Cheung, S. S. \& Kamath, C. (2004). Robust techniques for background subtraction in urban traffic video. IS\&T/SPIE's Symposium on Electronic Imaging, 881-892. https://doi.org/10.1117/12.526886

[14] Morris, B. T. \& Trivedi, M. M. (2008). Learning, modeling, and classification of vehicle track patterns from live video. IEEE Transactions on Intelligent Transportation Systems, 9(3), 425-437. https://doi.org/10.1109/TITS.2008.922970

[15] Çandar, C. \& Gerek, Ö. N. Yol üzerinde trafik ve araç durumunun video analiz yöntemleri ile incelenmesi. https://www.researchgate.net/profile/OmerGerek/publicatio $\mathrm{n} / 228400473$

[16] Öz， K. \& Görgünoğlu， S. (2016). Video Gözetim Sistemlerinde Anomali Tespiti Üzerine Bir Derleme. ElCezeri Journal of Science and Engineering, 3(3). https://doi.org/10.31202/ecjse.258578

[17] Shantaiya, S., Verma, K., \& Mehta, K. (2013). A Survey on Approaches of Object Detection.pdf. International Journal of Computer Applications, 65(18), 14-20.

[18] Sivaraman, S. \& Trivedi, M. M. (2013). Looking at vehicles on the road: A survey of vision-based vehicle detection, tracking, and behavior analysis. IEEE Transactions on Intelligent Transportation Systems, 14(4), 1773-1795. https://doi.org/10.1109/TITS.2013.2266661

[19] Faruk Ertuğrul, Ö. \& Kaya, Y. (2014). A detailed analysis on extreme learning machine and novel approaches based on ELM. American Journal of Computer Science and Engineering, 1(5), 43-50. Retrieved from http://www.openscienceonline.com/journal/ajcse

[20] Ertugrul, Ö. F. (2016). Forecasting electricity load by a novel recurrent extreme learning machines approach. International Journal of Electrical Power and Energy Systems, 78, 429435. https://doi.org/10.1016/j.jiepes.2015.12.006

[21] Kaya, Y., Ertuğrul, Ö. F., \& Tekin, R. (2014). An expert spam detection system based on extreme learning machine. Computer Science and Applications, 1(2), 132-137.

[22] Ertugrul, Ö.F., \& Kaya, Y. (2016). Smart city planning by estimating energy efficiency of buildings by extreme learning machine. In $4^{\text {th }}$ International Istanbul Smart Grid Congress and Fair, ICSG 2016. https://doi.org/10.1109/SGCF.2016.7492420

[23] Takma, Ç., Atıl, H., \& Aksakal, V. (2012). Comparison of multiple linear regression and artificial neural network models goodness of fit to lactation milk yields. Kafkas Üniversitesi Veteriner Fakültesi Dergisi, 18(6), 941-944. https://doi.org/10.9775/kvfd.2012.6764

[24] Basharat, a, Gritai, a, \& Shah, M. (2008). Learning object motion patterns for anomaly detection and improved object detection. In Computer Vision and Pattern Recognition, CVPR 2008. IEEE Conference on, 1-8. https://doi.org/10.1109/CVPR.2008.4587510

[25] Zhu, Y., Nayak, N. M., \& Roy-Chowdhury, A. K. (2013). Context-Aware Activity Recognition and Anomaly 
Detection in Video. Selected Topics in Signal Processing,

IEEE Journal of, 7(1), 91-101.

https://doi.org/10.1109/JSTSP.2012.2234722

\section{Contact information:}

Yasin SÖNMEZ, Instructor

(Corresponding author)

Dicle University

Technical Sciences Vocational School

21280 Diyarbakır, Turkey

yasin.sonmez@dicle.edu.tr

Hüseyin KUTLU, Instructor

Adıyaman University

Besni Vocational School

02040 Adıyaman, Turkey

huseyinkutlu02@gmail.com

Engin AVCl, Professor

Firat University

Technology Faculty - Software engineering

23119 Elazığ, Turkey

enginavci@firat.edu.tr 